\title{
Clinical Conundrums in Management of Hypothyroidism in Critically Ill Geriatric Patients
}

\author{
Vishal Sehgal $^{1, *} ;$ Sukhminder Jit Singh Bajwa ${ }^{2}$; Rinku Sehgal ${ }^{3}$; Anurag Bajaj ${ }^{3}$ \\ ${ }^{1}$ The Commonwealth Medical College, Scranton, PA, USA \\ ${ }_{3}^{2}$ Department of Anaesthesiology and Intensive Care Medicine, Gian Sagar Medical College, Banur, Patiala, India \\ ${ }_{3}^{3}$ The Wright Center for Graduate Medical education, Scranton, PA, USA \\ *Corresponding author: Vishal Sehgal, Department of Medicine, The Common wealth Medical College, Scranton, PA, USA, E-mail: sehgalvishal@hotmail.com
}

Received: July 22, 2013; Revised: September 22, 2013; Accepted: September 24,2013

\begin{abstract}
Context: Articles in various international and national bibliographic indices were extensively searched with an emphasis on thyroid and hypothyroid disorders, hypothyroidism in elderly hospitalized patients, hypothyroidism in critically ill geriatric population, thyroxine in elderly hypothyroid, drug interactions and thyroid hormones, and thyroid functions in elderly.

Evidence acquisition: Entrez (including PubMed), NIH.gov, Medscape.com, WebMD.com, MedHelp.org, Search Medica, MD consult, yahoo.com, and google.com were searched. Manual search was performed on various textbooks of medicine, critical care, pharmacology, and endocrinology.

Results: Thyroid function tests in elderly hospitalized patients must be interpreted with circumspection. The elderly are often exposed to high iodide content and critical care settings. This may occur because of either decreased iodine excretion or very high intake of iodine. This is especially true for elderly population with underlying acute or chronic kidney diseases or both. Amiodarone, with a very high iodine content, is also often used in this set of population. Moreover, other medications including iodinated contrast are often used in the critical care settings. These may affect different steps of thyroid hormone metabolism, and thereby complicate the interpretation of thyroid function tests.

Conclusions: The current review is aimed at analyzing and managing various clinical aspects of hypothyroidism in hospitalized elderly, and critically ill geriatric patients.
\end{abstract}

Keywords:Amiodarone; Kidney Failure; Chronic; Critical Illness; Hypothyroidism; Polypharmacy

\section{Context}

Thyroid disorders are the second most common endocrine disorders after diabetes mellitus type II (DM-II). Clinical presentation is often vague and nonspecific, and its diagnosis is often dependent on laboratory investigations. Hypothyroidism is sub-classified into three types based on laboratory studies. Primary hypothyroidism is characterized by high thyroid stimulating hormone (TSH) and low free thyroxine (T4) levels. Secondary hypothyroidism is defined as low free T4 and TSH, which is not appropriately increased. Subclinical hypothyroidism (SH) is defined as low normal free T4 and high TSH.

\section{Evidence Acquisition}

Hypothyroidism in geriatric and critically ill elderly patients can be a significant cause for higher morbidity and mortality rates. Hypothyroidism has high prevalence in geriatric population, and is five to eight times more common in women $(1,2)$. Its incidence is increased with aging. However, the literature estimation of hypothyroidism prevalence among the elderly exhibits a varied picture depending on the studied populations and the criteria to define it. Even in developed nations like the United States (US), the national health and nutrition examination survey (NHANESIII) estimated the prevalence of hypothyroidism approximately $4.6 \%$, (0.3\% as overt and $4.3 \%$ as SH2). These statistics convey a similar picture among different set-ups and points, with hypothyroid as the second most common endocrine disorder visited by clinicians in geriatric practice (3). Overall, in the US, hypothyroid affects more than 10 million people (4).

Challenges facing clinicians come from the invariably atypical clinical presentation of hypothyroidism. This clinical presentation in hospitalized elderly patients is associated with increased morbidity and mortality rates.

Implication for health policy/practice/research/medical education:

Successful management of hypothyroidism in the elderly is mainly dependent on considering body physiology with aging. It would be important to keep in mind underlying kidney diseases and medications, which might affect the metabolism of thyroid hormones in the body. If medications and underlying comorbidities be not carefully considered, it may lead to inappropriate use of THRT and resultant side effects for patients. In addition, polypharmacy and multiple comorbidities frequently seen in elderly, may pose clinical complications in the interpretation of thyroid function tests. A comprehensive understanding of the subject would help clinicians avoid mismanagement of this common clinical presentation.

Copyright (C) 2014, Research Institute For Endocrine Sciences and Iran Endocrine Society; Published by Kowsar Corp. This is an open-access article distributed under the terms of the Creative Commons Attribution License, which permits unrestricted use, distribution, and reproduction in any medium, provided the original work is properly cited. 
The endocrine emergencies exhibit higher morbidity and mortality rates in elderly patients who undergo thyroid surgical procedures (5-8).

\section{Results}

\subsection{Literature Search Strategies}

Articles in various international and national bibliographic indices were extensively searched with an emphasis on thyroid and hypothyroid disorders, hypothyroidism in elderly hospitalized patients, hypothyroidism in critically ill geriatric population, thyroxine in elderly hypothyroid, drug interactions and thyroid hormones, and thyroid functions in elderly. The emphasis was also on the epidemiology, pattern of clinical presentations, diagnostic modalities, therapeutic interventions and complications due to the hypothyroid disorders in elderly hospitalized patients. Drug-drug interactions as well as the side effects and drug interactions with thyroid hormones were also considered. Entrez (including PubMed), NIH.gov, Medscape.com, WebMD.com, MedHelp.org, Search Medica, MD consult, yahoo.com, and google.com were searched. Manual search was performed on various textbooks of medicine, critical care, pharmacology, and endocrinology.

\subsection{Hypothyroidism in Hospitalized Elderly: Etio- logical Factors}

According tothe literature, autoimmune thyroiditis is the most common cause of hypothyroidism among the elderly and younger persons. Among other causes, Iodine deficiency, radioiodine ablation, and surgery are significant causes of hypothyroidism in elderly hospitalized patients $(9,10)$. The incidence of Iodine deficiency related hypothyroidism is decreased in India due to various national initiatives on universal salt iodization (3).

\subsection{Clinical Epidemiology and Symptomatology of Hypothyroidism in Elderly}

Physiological variations between very old and moderately older patients are significant with changes with every passing decade.The symptomatology of hypothyroidism is less specific in elderly patients compared to younger generation, which poses diagnostic challenges to clinicians (Table 1). Moreover, hypothyroidism may often be masked by co-morbidities like DM-II (11). The difficulty in diagnosis is further exaggerated due to various drug interactions related to polypharmacy, which is quite common in this aging population (12). Acute onset of hypothyroidism and thyroid hormone deficiency of higher magnitude can possibly result in exacerbation of symptoms in a robust manner. However, symptoms can be better tolerated if hypothyroidism starts gradually. Both hypothyroidism and SH may be associated with a number of abnormalities which can be present in critically ill patients. These may include but not limited to cardiac, pulmonary, gastro-intestinal (GI), hematological, neurological, and metabolic manifestations $(13,14)$.

Table 1. Common Clinical Presentation of Hypothyroidism in Critically Ill Geriatric Population

\begin{tabular}{|l|l|}
\hline Systems & Clinical Manifestations \\
\hline Cardiac & Congestive heart failure \\
& Pericardial effusion \\
& Hypertension \\
\hline & Decreased cardiac output \\
& Angina \\
\hline Pulmonary & Decrease response to hypercapnia \\
& and hypoxia \\
\hline Macroglossia \\
\hline Hematologic & Hyperlipidemia \\
\hline Constipation \\
\hline
\end{tabular}

Hypothyroidism is a lower risk of atrial fibrillation as compared to a much higher risk in hyperthyroid patients which can directly affect the outcome in critically ill patients with hypothyroidism (15). The resultant reduced cardiac output can contribute to decreased functional cardio-respiratory reserve. Additionally, heart failure and angina may worsen in a patient with acute onset of hypothyroidism and pre-existing coronary artery disease (CAD).However, other cardiac manifestations of hypothyroidism in critical care settings may include but not limited to pericardial effusion, hypertension (HTN) and hyperlipidemia (HLP). It is proven that thyroid hormone replacement therapy (THRT) is beneficial in such critically ill patients (16). Hypothyroidism, both overt and subclinical, are definitely associated with post-prandial HLP; and metabolic syndrome is clarified $(17,18)$.

Hypothyroidism can also cause weakness of the respiratory muscles and a reduced pulmonary response to hypercapnia and hypoxia. However, shortness of breath seen in hypothyroidism is due to multiple etiologies related to cardiac and pulmonary tissues, while structural abnormalities such as macroglossia may contribute to 
sleep apnea. These abnormalities could potentially be reversed by appropriate thyroid hormone replacement therapy (THRT) (18).

Constipation is the most common GI complication occurred in hypothyroidism, while frequent association of nonalcoholic fatty liver disease and hypothyroidism is often a matter of clinical concern (19). Among autoimmune disorders, pernicious anemia is associated with $10 \%$ of the cases of autoimmune thyroiditis (20). The frequency of anemia of chronic disease is almost equally distributed in both $\mathrm{SH}$ and overt hypothyroidism (21).

Central nervous system involvement in hypothyroidism include complex mental status changes, as the main clinical presentation of neurological manifestations(22). Characteristically, Hashimoto's encephalopathy (HE) which presents as complex mental changes is immunemediated and not a direct manifestation of hypothyroidism. However, the diagnosis is established only by excluding other causes of mental status changes, also there are some evidences of positive antithyroid antibodies levels. Neurological manifestations of hypothyroidism in critically ill elderly usually present as mental status changes and HE though rare must be in the list of differentials (22). Myxedema coma is an uncommon diagnosis in the ICU, but can be seen when severe hypothyroidism is complicated by infection, trauma, hypothermia narcotics useand benzodiazepines $(5,23)$. The typical clinical presentation is characterized by a comatose state withpossible association of hyponatremia, hypothermia and hypercapnia.

Hyperthyroidism incidence and levels of antithyroid antibodies increase with aging (24). Due to gradual onset of hyperthyroidism, the classical clinical features of heat intolerance, tachycardia, sweating and tremor may not be seen and are often absent in critically ill patients. Though of limited clinical significance in ICU, subtle signs such as fatigue, muscle weakness, weight loss and atrial arrhythmias are probably present in the patient history (25).

\subsection{Thyroid Functions in Hospitalized Geriatric Pa- tients}

Total or free thyroxine concentrations do not show any marked changes in elderly, because both thyroxine clearance and production decrease with aging $(26,27)$. At molecular level, such changes are associated with decreased nocturnal pulses of TSH secretion which counterbalances the decreased clearance of thyroxine in the elderly (28). The complicated clinical profile of critically ill geriatric patients mandates a dose reduction and initiation of lower doses of thyroxine supplementation with simulta- neous monitoring of TSH levels in spite of wider normal range of TSH in the elderly $(29,30)$. A number of studies have sought to determine whether biochemical diagnosis of thyroid disorders in the elderly may be confounded by age-related changes in thyroid function. It has also been observed that most elderly people with $\mathrm{SH}$ revert to euthyroid state, but higher the TSH levels, more is the possibility of developing overt hypothyroidism (31). TSH within the upper or lower end of normal range is a strong predictor for the possible development of hypo and hyperthyroidism (32). However, low total thyroxine (TT4) and high TSH are strongly associated with poor clinical prognosis in elderly hospitalized patients (33).

\subsection{Drug Interactions With Thyroid Hormones}

Some of the drugs commonly used in clinical practice may affect the metabolic pathway of thyroid hormones. Drug interactions could potentially affect different steps in the metabolism of thyroid hormones. Elderly usually have multiple co-morbidities and are frequently on polypharmacy (34). This increases the possibility of altered drug metabolism and drug interactions. Depending on the medication class or underlying pathology, druginduced hyperthyroidism, hypothyroidism or change in thyroid function profile may be seen (Figures 1 and 2).

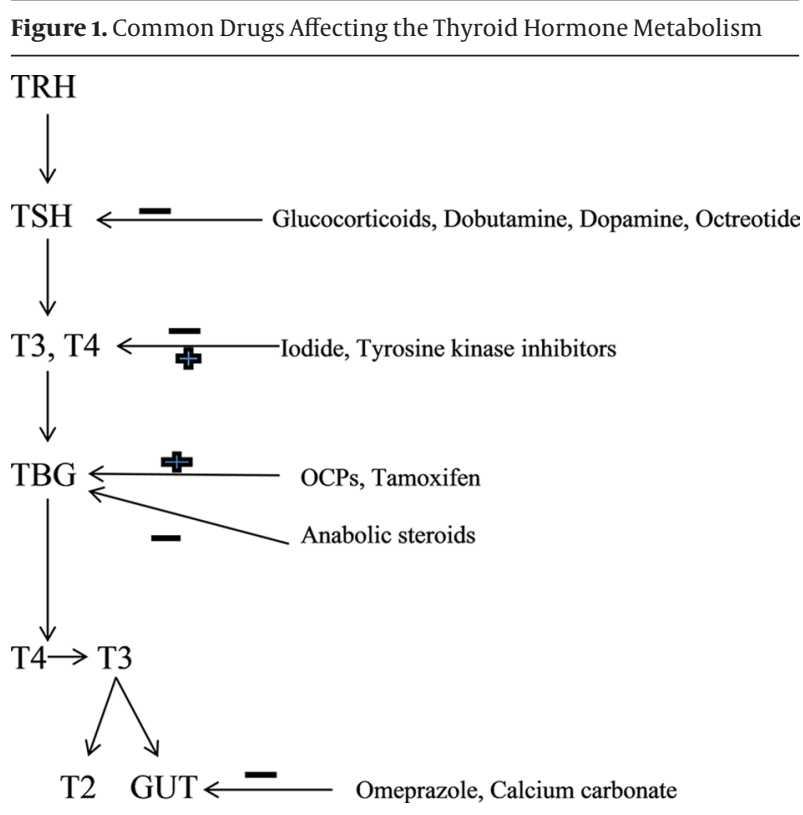

TRH, Thyrotropin Releasing Hormone; TSH, Thyrotropin; T3, Triiodothyronine; T4, Throxine; T2, Diiodothyronine. Drugs may either inhibit (-) or stimulate $(+)$ various steps in the metabolism of thyroid hormones. Omeprazole inhibits reabsorption of T3 and T4 from the intestine (GUT) 
Figure 2. Suppression of T2 and T3 Production by Inhibition of 5-Monodeiodinase Amiodarone, High-dose Steroids, Beta Blockers, Iodinated Contrast, Propylthiouracil, Steroids, Sepsis and NTIS

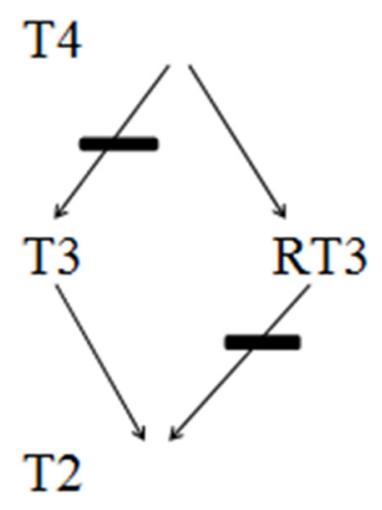

T3, Triiodothyronine; T4,Thyroxine; T2, Diiodothyronine; RT3, Reverse T3

In critical care setting, drugs that commonly affect TSH secretion may include but not limited to glucocorticoids (more than $20 \mathrm{mg}$ per day of Prednisone or its equivalents), Dobutamine, Dopamine, Octreotide and others. Iodine and Iodine containing drugs such as Amiodarone and contrast agents can potentially cause hyperthyroidism, especially in patients with underlying thyroid adenoma. Wolff-Chaikoff (WC) effect seen in such patients is caused by decreased thyroid hormone synthesis due to decreased oxidation of Iodide resulting from higher doses of Iodine. Antipsychotic medication Lithium is another drug with a potential to cause hypothyroidism. It has also been observed that even therapeutic concentration of Lithium can cause mild hypothyroidism in one-fifth of patients. Development of over thypothyroidism primarily necessitates treatment of hypothyroidism rather than stopping the Lithium therapy. Such patients are likely to need titration of THRT on stoppage of lithium and thus should be closely followed. Tyrosine kinase inhibitors are often used in the treatment of GI and hematological malignancies. Hypothyroidism in such patients with previously normal thyroid isoften preceded by destructive thyroiditis and transient suppression of TSH. Commonly used drugs such as OCPs, Tamoxifen and Methadoneinterfere with thyroid hormone transport and metabolism, and can potentiallyraise TBG. Drugs that affect and interfere with GI absorption of thyroid hormones include bile acid resins, proton pump inhibitors and calcium carbonate (Figure 1).Increased metabolism of T4 and T3 can be potentially influenced by phenobarbital, rifampin, phenytoin and carbamazepine. All these pharmacological interactions necessitate a higher dose of THRT in elderly hospitalized patients. Caution has to be exercised in the usage of drugs such as Amiodarone, steroids and beta blockers in hospitalized elderly as they inhibit 5-monodeiodinase, and thereby cause decreased formation of T3 (Figure 2).

\subsection{Thyroid Function Tests in Critical Care Settings}

Iodide exposure in critical care settings is one of the key determinants of thyroid hormone dysfunction. Elderly are often exposed to iodide in the critical care settings with increasing use of imaging studies and medications containing high doses of iodide causing impaired iodide auto-regulation mechanisms. The decreased production of thyroid hormones secondary to decreased organification of iodide may lead to WC effect (35). As kidney is largely instrumental in iodide clearance by glomerular filtration, iodide tends to accumulate and may enhance WC effect in patients with renal failure possibly resulting in higher frequency of goiter and hypothyroidism in such patients $(36,37)$.

Sepsis is commonly encountered in critically ill patients and can increase cytokine levels in the body, which could potentially suppress 5 -monodeiodinases (38). The resulting molecular interactions can decrease conversion of T4 to T3 and a decreased release of thyrotrophic releasing hormone (TRH) from the hypothalamus. This clinical presentation is often termed as non-thyroid illness syndrome (NTIS),and also known as sick euthyroid syndrome. This clinical entity is characterized by reverse T3 (RT3) and T4elevation due to inhibition of 5-monodeiodinasesand 5-monodeiodinases, respectively (Figure 2).RT3 may need to be measured to distinguish central hypothyroidism from NTIS, as RT3 levels are high in patients with NTIS except those with renal failure (36). Overall, these endocrinological changes are natural adaptation to reduce the catabolism in the body in diseased states. These lower levels of T3 hormones are highly associated with increased cardiovascular morbidity and mortality rates in uremic patients (39).

Increased incidence of head and neck cancers is also associated with aging. Such cancers are not usually treated with surgical procedures, and may require radiotherapy for therapeutic and palliative purposes. However, a prolonged exposure to radiotherapy can potentially damage the thyroid gland resulting in hypo or hyperthyroidism, which often requires screening when such patients are hospitalized specifically in ICU (40).

\subsection{Drugs Affecting Thyroid Function in Critical Care Settings}

Amiodarone is rich in iodine (250 times the daily recommended dose of iodine),which are commonly prescribed in critical care settings in the elderly population to control atrial fibrillation (AF) (41). This excessively high dose of Iodine has the potential to cause hyperthyroidism or hypothyroidism depending on the clinical scenarios and geographical area of the patient population. In patients living in iodine deficiency areas it causes hyperthyroidism, and in iodine sufficient areas it can cause hypothyroidism (42). Usually during the early phase of therapy, a decrease in T3 and an increase in RT3 and T4 may be 
observed (Figure 2). T4 and RT3 remain slightly elevated and T3 attains the normal levels after a prolonged period of therapy. A higher incidence of amiodarone-induced hypothyroidism (WC effect) may be observed in patients with underlying autoimmune thyroiditis. On the contrary, Jod-Basedow effect (JB effect) may be observed in patients with underlying goiter or latent Graves' disease, as the increased availability of iodide substrate can lead to secondary hyperthyroidism. Amiodarone could potentially block 5-monodeiodinasein younger patients. It causes hyperthyroidism in younger patients, while older patients tend to get $\mathrm{SH}$ or overt hypothyroidism $(43,44)$. Most of the patients with no underlying thyroid problems remain euthyroid during amiodarone therapy. However, one study showed that 5 percent of patients developed overt hypothyroidism (TSH $>10 \mathrm{mU} / \mathrm{L}$ ), and about 25 percent of patients developed SH (TSH 4.5-10) (45). The symptoms of hypothyroidism may be observed even after the treatment with amiodarone has been stopped, which can be explained based on lipophilic nature and its long half-life. As such, a periodic monitoring of TSH in patients treated with amiodarone is essential even in critical care set-ups.

Dopamine agonists and glucocorticoids commonly used in hospitalized patients may lead to transient central hypothyroidism, and a potential to worsen pre-existing hypothyroidism $(46,47)$.

\subsection{Management of Hypothyroidism in Hospital- ized Geriatric Patients}

Meticulous analysis and scrutiny of drugs and underlying pathology is needed prior to initiating THRT in the inpatient settings. Unwarranted use of THRT in elderly may lead to cardiac arrhythmias with higher chances of developing $\mathrm{AF}$ (48). In the critical care settings, geriatric patients may have multivariate clinical presentations with hypothyroidism, hyperthyroidism, SH, NTIS, JB effect, WC effect and HE which can cause diagnostic and therapeutic difficulties.

THRT should be started with caution in elderly, as most of them have underlying CAD. Once cardiovascular tolerance of a starting dose has been assessed, most experts recommend gradually increasing the daily dosage by 12.5-25 microgram ( $\mathrm{mcg}$ ) every four to six weeks. Annual TSH checking may not be necessary in all patients (49).

In non-elderly population, $\mathrm{SH}$ treatment leads to better control of cardiac problems (50). In contrast, studies of geriatric populations have not consistently shown similar benefits in treating SH (51). The distribution of TSH among population progressively shifts higher with aging (52). In elderly, there is a higher risk of heart failure, when TSH is higher than $10 \mathrm{mU} / \mathrm{L}$ (53). THRT may be considered for older people with anti thyroperoxidase antibody and TSH levels more than $10 \mathrm{mU} / \mathrm{L}(54,55)$. SH treatment has been associated with delayed progression of chronic kidney disease in elderly females $(56,57)$. There is no associa- tion between untreated persistent $\mathrm{SH}$ and progression of CHF, CAD or mortality, if TSH is less than 10Miu/L in geriatric population $(58,59)$.TSH levels in these patients may be normalized over the time (60).

NTIS is frequently seen in hospitalized elderly. There is no benefit in treating the same with THRT (61). JB effect is seen in the setting of iodine supplementation with underlying hyperthyroid state, which can be effectively treated by holding the iodine supplements and starting beta-blockers (62). Antithyroid medications may be started to speed up recovery. As JB typically lasts for 1-18 months, TFT should be monitored every 4-6 weeks initially and then every 3 months thereafter. It is usually possible to stop methimazole after 6-12 months of use in hospital.

Iodine induced hypothyroidism is seen in elderly patients with underlying SH. These patients are unusually sensitive to inhibitory effect of iodide, and if there is a need for continued use of these drugs the underlying hypothyroidism should be treated. When HE is suspected, steroids would be needed along with treatment of the dysthyroid state.

The challenges increase manifold in elderly diabetic and obese patients with associated thyroid disorders (63, 64). A new challenge has been encountered in today's critical care setting, as many patients with AIDS are admitted with various endocrine disorders(65). Elderly are often on polypharmacy and thyroid medications may be lost during medication reconciliation in transition from oral to enteral route in the ICU (66). Multidisciplinary team approach would help in ensuring appropriate and cost effective treatment in critically ill geriatric patients with hypothyroidism.

\section{Acknowledgments}

No acknowledgements

\section{Authors' Contribution}

All authors contributed equally.

\section{Financial Disclosure}

We had no financial disclosure.

\section{Funding/Support}

There was no funding support.

\section{References}

1. Aoki Y, Belin RM, Clickner R, Jeffries R, Phillips L, Mahaffey KR. Serum TSH and total T4 in the United States population and their association with participant characteristics: National Health and Nutrition Examination Survey (NHANES 1999-2002). Thyroid. 2007;17(12):1211-23.

2. So M, MacIsaac RJ, Grossmann M. Hypothyroidism. Aust Fam Physician. 2012;41(8):556-62.

3. Marwaha RK, Tandon N, Ganie MA, Kanwar R, Sastry A, Garg MK, et al. Status of thyroid function in Indian adults: two decades af- 
ter universal salt iodization. J Assoc Physicians India. 2012;60:32-6.

4. Huber G, Staub JJ, Meier C, Mitrache C, Guglielmetti M, Huber P, et al. Prospective study of the spontaneous course of subclinical hypothyroidism: prognostic value of thyrotropin, thyroid reserve, and thyroid antibodies. JClin Endocrinol Metab. 2002;87(7):3221-6.

5. Bajwa SJ, Jindal R. Endocrine emergencies in critically ill patients: Challenges in diagnosis and management. Indian J Endocrinol Metab. 2012;16(5):722-7.

6. Bajwa SJ, Sehgal V. Anesthesia and thyroid surgery: The never ending challenges. Indian J Endocrinol Metab. 2013;17(2):228-34 .

7. Bajwa SJ, Sehgal V. Anesthetic management of primary hyperparathyroidism: A role rarely noticed and appreciated so far. Indian J Endocrinol Metab. 2013;17(2):235-9.

8. Mingote E, Merono T, Rujelman R, Marquez A, Fossati P, Gurfinkiel M, et al. High TSH and low T4 as prognostic markers in older patients. Hormones (Athens). 2012;11(3):350-5.

9. Gervasi R, Orlando G, Lerose MA, Amato B, Docimo G, Zeppa P, et al. Thyroid surgery in geriatric patients: a literature review. $B M C$ Surg. 2012;12 Suppl 1:S16.

10. Gessl A, Lemmens-Gruber R, Kautzky-Willer A. Thyroid disorders. Handb Exp Pharmacol. 2012;(214):361-86.

11. Tamez-Perez HE, Martinez E, Quintanilla-Flores DL, Tamez-Pena AL, Gutierrez-Hermosillo H, Diaz de Leon-Gonzalez E. The rate of primary hypothyroidism in diabetic patients is greater than in the non-diabetic population. An observational study. Med Clin (Barc). 2012;138(11):475-7.

12. Martin CM. Thyroid dysfunction and the elderly patient: a primer for pharmacists. Consult Pharm. 2012;27(10):682-8.

13. Kasatkina SG, Panova TN. [Impact of replacement therapy for subclinical hypothyroidism on cardiovascular risk markers in patients with type 2 diabetes mellitus]. Ter Arkh. 2012;84(11):4750.

14. Mitu F, Cojocaru E, Tamba B, Leon MM. [Prevalence of cardiovascular diseases in patients with hypothyroidism]. Rev Med Chir Soc Med Nat Iasi. 2012;116(2):413-8.

15. Selmer C, Olesen JB, Hansen ML, Lindhardsen J, Olsen AM, Madsen JC, et al. The spectrum of thyroid disease and risk of new onset atrial fibrillation: a large population cohort study. BMJ. 2012;345.

16. Stuijver DJ, van Zaane B, Gerdes VE, Stroes ES. [Screening for thyroid dysfunction in dyslipidaemia patients]. Ned Tijdschr Geneeskd. 2012;156(2).

17. Arikan S, Bahceci M, Tuzcu A, Celik F, Gokalp D. Postprandial hyperlipidemia in overt and subclinical hypothyroidism. Eur J Intern Med. 2012;23(6):e141-5.

18. Waring AC, Rodondi N, Harrison S, Kanaya AM, Simonsick EM, Miljkovic I, et al. Thyroid function and prevalent and incident metabolic syndrome in older adults: the Health, Ageing and Body Composition Study. Clin Endocrinol (Oxf). 2012;76(6):911-8.

19. Pagadala MR, Zein CO, Dasarathy S, Yerian LM, Lopez R, McCullough AJ. Prevalence of hypothyroidism in nonalcoholic fatty liver disease. Dig Dis Sci. 2012;57(2):528-34.

20. Li X, Sundquist J, Sundquist K. Subsequent risks of Parkinson disease in patients with autoimmune and related disorders: a nationwide epidemiological study from Sweden. Neurodegener Dis. 2012;10(1-4):277-84

21. Erdogan M, Kosenli A, Ganidagli S, Kulaksizoglu M. Characteristics of anemia in subclinical and overt hypothyroid patients. Endocr J. 2012;59(3):213-20.

22. Chavanne D, Constans T, Petit A, Mondon K, Hommet C. Hypothyroidism and cognition in the elderly. Geriatr Psychol Neuropsychiatr Vieil. 2011;9(4):477-89.

23. Ringel MD. Management of hypothyroidism and hyperthyroidism in the intensive care unit. Crit Care Clin. 2001;17(1):59-74.

24. Hollowell JG, Staehling NW, Flanders WD, Hannon WH, Gunter EW, Spencer CA, et al. Serum TSH, T(4), and thyroid antibodies in the United States population (1988 to 1994): National Health and Nutrition Examination Survey (NHANES III). J Clin Endocrinol Metab. 2002;87(2):489-99.

25. Frost L, Vestergaard P, Mosekilde L. Hyperthyroidism and risk of atrial fibrillation or flutter: a population-based study. Arch Intern Med. 2004;164(15):1675-8.
26. Oddie TH, Meade JJ, Fisher DA. An analysis of published data on thyroxine turnover in human subjects. J Clin Endocrinol Metab. 1966;26(4):425-36.

27. Runnels BL, Garry PJ, Hunt WC, Standefer JC. Thyroid function in a healthy elderly population: implications for clinical evaluation. J Gerontol.1991;46(1):B39-44.

28. Greenspan SL, Klibanski A, Rowe JW, Elahi D. Age-related alterations in pulsatile secretion of TSH: role of dopaminergic regulation. Am J Physiol.1991;260(3 Pt 1):E486-91.

29. Canaris GJ, Manowitz NR, Mayor G, Ridgway EC. The Colorado thyroid disease prevalence study. Arch Intern Med. 2000;160(4):52634.

30. Gaffney GW, Gregerman RI, Shock NW. Relationship of age to the thyroidal accumulation, renal excretion and distribution of radioiodide in euthyroid man. J Clin Endocrinol Metab. 1962;22:78494.

31. Somwaru LL, Rariy CM, Arnold AM, Cappola AR. The natural history of subclinical hypothyroidism in the elderly: the cardiovascular health study. J Clin Endocrinol Metab. 2012;97(6):1962-9.

32. Asvold BO, Vatten LJ, Midthjell K, Bjoro T. Serum TSH within the reference range as a predictor of future hypothyroidism and hyperthyroidism: 11-year follow-up of the HUNT Study in Norway. $J$ Clin Endocrinol Metab. 2012;97(1):93-9.

33. Ilias I, Stamoulis K, Armaganidis A, Lyberopoulos P, Tzanela M, Orfanos $\mathrm{S}$, et al. Contribution of endocrine parameters in predicting outcome of multiple trauma patients in an intensive care unit. Hormones (Athens). 2007;6(3):218-26.

34. Bajwa SJ, Panda A. Alternative medicine and anesthesia: Implications and considerations in daily practice. Ayu. 2012;33(4):475-80.

35. Wolff J, Chaikoff IL. Plasma inorganic iodide as a homeostatic regulator of thyroid function. J Biol Chem.1948;174(2):555-64.

36. Bajwa SS, Kwatra IS. Nutritional needs and dietary modifications in patients on dialysis and chronic kidney disease. J Med Nutr Nutraceut. 2013;2(1):46.

37. Kaptein EM. Thyroid hormone metabolism and thyroid diseases in chronic renal failure. Endocr Rev. 1996;17(1):45-63.

38. van der Poll T, Romijn JA, Wiersinga WM, Sauerwein HP. Tumor necrosis factor: a putative mediator of the sick euthyroid syndrome in man. J Clin Endocrinol Metab.1990;71(6):1567-72.

39. Meuwese CL, Dekker FW, Lindholm B, Qureshi AR, Heimburger $\mathrm{O}$, Barany $\mathrm{P}$, et al. Baseline levels and trimestral variation of triiodothyronine and thyroxine and their association with mortality in maintenance hemodialysis patients. Clin J Am Soc Nephrol. 2012;7(1):131-8.

40. Bakhshandeh M, Hashemi B, Mahdavi SR, Nikoofar A, Edraki HR, Kazemnejad A. Evaluation of thyroid disorders during head-andneck radiotherapy by using functional analysis and ultrasonography. Int J Radiat Oncol Biol Phys. 2012;83(1):198-203.

41. Basaria S, Cooper DS. Amiodarone and the thyroid. Am J Med. 2005;118(7):706-14.

42. Trip MD, Wiersinga W, Plomp TA. Incidence, predictability, and pathogenesis of amiodarone-induced thyrotoxicosis and hypothyroidism. Am J Med.1991;91(5):507-11.

43. Triggiani V, Iacoviello M, Monzani F, Puzzovivo A, Guida P, Forleo $\mathrm{C}$, et al. Incidence and prevalence of hypothyroidism in patients affected by chronic heart failure: role of amiodarone. Endocr Metab Immune Disord Drug Targets. 2012;12(1):86-94.

44. Zosin I, Balas M. Amiodarone-induced thyroid dysfunction in an iodine-replete area: epidemiological and clinical data. Endokrynol Pol. 2012;63(1):2-9.

45. Batcher EL, Tang XC, Singh BN, Singh SN, Reda DJ, Hershman JM et al. Thyroid function abnormalities during amiodarone therapy for persistent atrial fibrillation. Am J Med. 2007;120(10):880-5.

46. Haugen BR. Drugs that suppress TSH or cause central hypothyroidism. Best Pract Res Clin Endocrinol Metab. 2009;23(6):793-800.

47. Thomas Z, Bandali F, McCowen K, Malhotra A. Drug-induced endocrine disorders in the intensive care unit. Crit Care Med. 2010;38(6 Suppl):S219-30.

48. Somwaru LL, Arnold AM, Joshi N, Fried LP, Cappola AR. High frequency of and factors associated with thyroid hormone overreplacement and under-replacement in men and women aged 65 and over. J Clin Endocrinol Metab. 2009;94(4):1342-5. 
49. Pecina J, Bernard M, Furst J, Rohrer J. Hypothyroidism management: is an annual check of TSH level always necessary? J Fam Pract. 2012;61(10):E1-5.

50. Rakhmatullov FK, Klimova SV, Rudakova LE, Pchelinsteva SA, Rakhmatullov AF, Khodakova AI. [The influence of subclinical thyroid dysfunction on cardiac hemodynamic and electrophysiological parameters in patients with coronary heart disease and paroxysmal atrial fibrillation]. Klin Med (Mosk). 2012;90(10):31-4.

51. Bensenor IM, Olmos RD, Lotufo PA. Hypothyroidism in the elderly: diagnosis and management. Clin Interv Aging. 2012;7:97-111.

52. Surks MI, Hollowell JG. Age-specific distribution of serum thyrotropin and antithyroid antibodies in the US population: implications for the prevalence of subclinical hypothyroidism. J Clin Endocrinol Metab. 2007;92(12):4575-82.

53. Gencer B, Collet TH, Virgini V, Bauer DC, Gussekloo J, Cappola AR, et al. Subclinical thyroid dysfunction and the risk of heart failure events: an individual participant data analysis from 6 prospective cohorts. Circulation. 2012;126(9):1040-9.

54. Goichot B, Luca F. [Subclinical thyroid dysfunction]. Presse Med. 2011;40(12 Pt1):1132-40.

55. Wiersinga WM. [Cardiovascular risks in patients with subclinical thyroid dysfunction]. Ned Tijdschr Geneeskd. 2012;156(49).

56. Saini V, Yadav A, Arora MK, Arora S, Singh R, Bhattacharjee J. Correlation of creatinine with TSH levels in overt hypothyroidism - a requirement for monitoring of renal function in hypothyroid patients? Clin Biochem. 2012;45(3):212-4.

57. Shin DH, Lee MJ, Kim SJ, Oh HJ, Kim HR, Han JH, et al. Preservation of renal function by thyroid hormone replacement therapy in chronic kidney disease patients with subclinical hypothyroidism. J Clin Endocrinol Metab. 2012;97(8):2732-40.
58. Biondi B. Natural history, diagnosis and management of subclinical thyroid dysfunction. Best Pract Res Clin Endocrinol Metab. 2012;26(4):431-46.

59. Diez JJ, Iglesias P, Burman KD. Spontaneous normalization of thyrotropin concentrations in patients with subclinical hypothyroidism. J Clin Endocrinol Metab. 2005;90(7):4124-7.

60. Surks MI, Ortiz E, Daniels GH, Sawin CT, Col NF, Cobin RH, et al. Subclinical thyroid disease: scientific review and guidelines for diagnosis and management. JAMA. 2004;291(2):228-38.

61. Bello G, Ceaichisciuc I, Silva S, Antonelli M. The role of thyroid dysfunction in the critically ill: a review of the literature. Minerva Anestesiol. 2010;76(11):919-28.

62. Bahn Chair RS, Burch HB, Cooper DS, Garber JR, Greenlee MC, Klein I, et al. Hyperthyroidism and other causes of thyrotoxicosis: management guidelines of the American Thyroid Association and American Association of Clinical Endocrinologists. Thyroid. 2011;21(6):593-646.

63. Bajwa SJ, Kalra S. Diabeto-anaesthesia: A subspecialty needing endocrine introspection. Indian J Anaesth. 2012;56(6):513-7.

64. Bajwa SJ, Sehgal V, Bajwa SK. Clinical and critical care concerns in severely ill obese patient. Indian J Endocrinol Metab. 2012;16(5):740-8.

65. Bajwa SJ, Kulshrestha A. The potential anesthetic threats, challenges and intensive care considerations in patients with HIV infection. J Pharm Bioallied Sci. 2013;5(1):10-6.

66. Barrett NA, Jones A, Whiteley C, Yassin S, McKenzie CA. Management of long-term hypothyroidism: a potential marker of quality of medicines reconciliation in the intensive care unit. Int $J$ Pharm Pract. 2012;20(5):303-6. 